\section{CVIA}

ORIGINAL ARTICLE

pISSN 2508-707X / eISSN 2508-7088 https://doi.org/10.22468/cvia.2019.00052 CVIA 2019;3(3):61-67

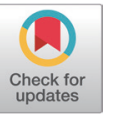

\title{
Volumetric Severity Assessment of Ebstein Anomaly Using Three-Dimensional Cardiac CT: A Feasibility Study
}

\author{
Hyun Woo Goo \\ Department of Radiology and Research Institute of Radiology, University of Ulsan \\ College of Medicine, Asan Medical Center, Seoul, Korea
}

\begin{abstract}
Objective: The purpose of this study was to demonstrate the feasibility of using cardiac CT to perform volumetric severity assessment of the Ebstein anomaly.

Materials and Methods: Six children with Ebstein anomaly were included. We used cardiac CT to measure the delineated tricuspid valve, streak artifacts, and contrast enhancement heterogeneity. Cardiac CT data were used to quantify the volumes of all of the cardiac chambers, including the atrial and functional right ventricle (RV). In addition, the functional RV (fRV) fraction, fRV/left ventricle (LV) volume ratio, and total right/left-volume index were calculated. The volume-based CT severity index was compared using area-based echocardiographic and CT severity indices.

Results: All of the patients had grade 3 or 4 extent of delineation of the malformed tricuspid valve and streak artifacts. In one case, the right atrium had image noise [126.7 Hounsfield units $(\mathrm{HU})$ ] that was $>50 \mathrm{HU}$, while all others demonstrated image noise $<50 \mathrm{HU}$. This image quality was appropriate to make all of the volumetric measurements. The RV volumes were enlarged in $83.3 \%(5 / 6)$ of the cases. The fRV fraction was $60.8-61.5 \%$. The fRV/LV volume ratio and total right/left-volume index were $2.0 \pm 1.4$ and $3.5 \pm 1.5$, respectively. There was a smaller difference $(0.2 \pm 0.1)$ between the area-based and volume-based CT severity indices than there was in other comparisons. These parameters also had a higher concordance rate $(83.3 \%, 5 / 6)$ in the severity grades.
\end{abstract}

Conclusion: Volumetric severity assessment using cardiac CT is feasible in Ebstein anomaly, and may be useful to characterize the disease's diverse phenotypes.

Key words Cardiac imaging technique - Ebstein anomaly · Multidetector computed tomography $\cdot$ Severity of illness index $\cdot$ Imaging, three-dimensional $\cdot$ Ventricular function.

Received: April 11, 2019

Al, May 7, 2019

Corresponding author Hyun Woo Goo, MD, PhD

Department of Radiology and Research Institute of Radiology, University of Ulsan College of Medicine, Asan Medical Center, 88 Olympic-ro 43-gil, Songpa-gu, Seoul 05505, Korea

Tel: 82-2-3010-4388

Fax: 82-2-476-0090

E-mail: ghw68@hanmail.net

\section{INTRODUCTION}

Ebstein anomaly is a rare congenital heart disease that accounts for $<1 \%$ of all congenital heart diseases. It is characterized by a congenital malformation of the tricuspid valve and the right ventricle (RV) [1]. Carpentier and Celermajer classifications, which are based on echocardiographic findings, have been used to predict the clinical outcomes in Ebstein anomaly [1] and to determine the surgical options according to its severity [2].

(a) This is an Open Access article distributed under the terms of the Creative Commons Attribution Non-Commercial License (https://creativecommons.org/licenses/bync/4.0) which permits unrestricted non-commercial use, distribution, and reproduction in any medium, provided the original work is properly cited.
However, echocardiography cannot provide an accurate volumetric severity index (SI). Cardiac MRI has also been used to quantify ventricular volumes and function in Ebstein anomaly. However, cine MRI is limited by its ability to accurately segment the malformed tricuspid valve and RV endocardial borders because of its two-dimensional (2D) nature [3-6].

Cardiac MRI is generally limited by its long examination time, which often requires conscious sedation or general anesthesia. In addition, the threshold-based segmentation results of threedimensional (3D) whole-heart MRI data are often influenced by inhomogeneous signal intensity and imaging artifacts [7]. In contrast, cardiac CT is increasingly used to evaluate congenital 
heart disease because of its short examination time, high image quality, and wide availability. Cardiac CT is not only useful for morphologic evaluation, but also ventricular function analysis $[8,9]$. The concerns regarding radiation risks from cardiac CT have dramatically diminished with the optimized low-dose CT protocols [10-12]. Volumetric assessments using high-resolution 3D cardiac CT may be advantageous over the conventional cardiac MRI method in congenital heart disease, and are frequently used at our institution [9]. However, no prior studies have evaluated these volumetric assessments in patients with Ebstein anomaly. The lack of associated literature is probably due to the technically challenging segmentation of the malformed tricuspid valve and the rarity of the anomaly. Therefore, this retrospective study was performed to demonstrate the feasibility of volumetric severity assessment of the Ebstein anomaly using 3D cardiac CT.

\section{MATERIALS AND METHODS}

This retrospective study was approved by the local Institutional Review Board. The requirement for informed consent was waived.

\section{Study population}

Between December 2010 and December 2016, six consecutive children with Ebstein anomaly (median age 5.2 years, age range 4 days -14 years; male:female $=1: 1$ ) who underwent cardiac CT examinations (to evaluate cardiac function) were included. Four of the six children had unrepaired Ebstein anomalies. The remaining two underwent surgical procedures before the cardiac CT examination. These procedures included tricuspid valvuloplasty with pulsatile bidirectional cavopulmonary shunt $(\mathrm{n}=1)$, and a central shunt and division of a patent ductus arteriosus $(\mathrm{n}=1)$. The body surface area at the cardiac CT examination (median $0.7 \mathrm{~m}^{2}$, range $0.2-1.8 \mathrm{~m}^{2}$ ) was used to normalize the measured CT values.

\section{Cardiac CT}

Cardiac CT was obtained using a second-generation dualsource scanner (SOMATOM Definition Flash; Siemens Healthineers, Forchheim, Germany). The scanning parameters were as follows: $2 \times 64 \times 0.6 \mathrm{~mm}$ slices with the $\mathrm{z}$-flying focal spot technique, $0.28 \mathrm{~s}$ gantry rotation time, $75 \mathrm{~ms}$ temporal resolution, $0.75 \mathrm{~mm}$ slice width, and $0.4-\mathrm{mm}$ reconstruction interval. The combined tube current modulation (CARE Dose 4D; Siemens Healthineers) was used in all of the cardiac CT examinations to reduce the radiation dose [13]. According to the optimized protocols for cardiac function assessment at our institution [14], electrocardiography (ECG)-synchronized CT scanning was performed using three different techniques depending on the child's age and cooperativity as follows: breath-hold retrospectively ECG-gated spiral scan was acquired in three old children; free-breathing combined prospectively respiratory- and ECGtriggered sequential scan in one infant; and free-breathing prospectively ECG-triggered sequential scan in two newborns. In addition to an aggressive version of the ECG-controlled tube current modulation (MinDose; Siemens Healthineers), we also used the so-called biphasic chest pain protocol (using half of the tube current outside the heart) to reduce the radiation dose of the retrospectively ECG-gated spiral scan [15]. The body sizeadapted radiation dose in prospectively ECG-triggered sequential scanning was estimated from the cross-sectional body area, and the mean body density of each patient [16]. In combined triggering, prospective respiratory triggering (AZ-733V; Anzai Medical Co., Tokyo, Japan) was added to the ECG-triggered sequential scan in order to reduce respiratory motion artifacts [17]. Low tube voltage was used to maximize the iodine contrast-tonoise ratio and radiation dose-saving effect: $70 \mathrm{kV}$ in three children, $80 \mathrm{kV}$ in two, and $100 \mathrm{kV}$ in one. An iodinated contrast agent (Iomeron 400, iomeprol $400 \mathrm{mg} \mathrm{I} / \mathrm{mL}$; Bracco Imaging SpA, Milan, Italy; $1.5-2.0 \mathrm{~mL} / \mathrm{kg}$ ) was administered intravenously using a tri-phasic or quadri-phasic injection protocol at an injection rate of $0.3-3.0 \mathrm{~mL} / \mathrm{s}$. The scan delay time was determined by a bolus tracking technique with a trigger threshold of 150 Hounsfield units (HU) in the left ventricular cavity. The dose-length product was based on a 32-cm-sized phantom. The effective dose was based on a dose-length product and age, sex, and tube voltage-specific conversion factors [18]. These were $197.1 \pm 206.5 \mathrm{mGy} \cdot \mathrm{cm}$ and $3.9 \pm 3.2 \mathrm{mSv}$, respectively.

\section{CT image quality evaluation}

In order to evaluate whether the cardiac CT images were high enough image quality to quantify the cardiac chambers, we graded the images on axial, coronal, and sagittal slices. We also graded the degree of heterogeneous contrast enhancement in the cardiac chambers, which were presented as a standard deviation of the measured attenuations. These measurements were made by placing a large region of interest in each cardiac chamber on the four-chamber CT images. The following volumes and measurements were made: ventricular and atrial volumes; the extent of the delineated tricuspid valve (grade 1: $\leq 25 \%$ of the valve; grade 2: $>25 \%$ and $\leq 50 \%$; grade $3:>50 \%$ and $\leq 75 \%$; grade 4: $>75 \%$ ); and the extent of streak artifacts from undiluted contrast agent in cardiac chambers (grade 1: severe artifacts degrading $>75 \%$ of a cardiac chamber; grade 2: moderate artifacts degrading $>50 \%$ and $\leq 75 \%$; grade 3 : mild artifacts degrading $>25 \%$ and $\leq 50 \%$; grade 4 : minimal artifacts degrading $\leq 25 \%$ ). Grade 3 or 4 reflected sufficiently high image quality (Fig. 1A-C). Image noise $>50 \mathrm{HU}$ had the potential to degrade the volume quantification. 


\section{CT volumetric quantification}

A commercially available workstation (Advantage Windows 4.6; GE Healthcare, Milwaukee, WI, USA) was used for volumetric quantification. A semiautomatic 3D threshold-based segmentation method, in which the papillary muscles and trabeculations were excluded from the ventricular cavity, was used to quantify the ventricular volumes. A segmentation threshold separating the interventricular septal myocardium from the adjacent ventricular blood was regarded to be optimal [9]. The valve and venoatrial planes were manually adjusted. The right ventricular cavity was further divided into the atrialized RV (aRV) and the functional RV (fRV) along the malformed tricuspid valve planes. The volumes of these two RV parts were quantified separately (Fig. 1D). In addition, the left ventricle (LV), right atrial, and left atrial volumes were similarly quantified (Fig. 1E-G). The preliminary volumetric segmentation was performed by a radiology technologist. The final result was confirmed by a radiologist with 19 years of experience in cardiac CT.

The ejection fraction $(\mathrm{EF})$ was calculated as follows: $\mathrm{EF}(\%)=$ [end-diastolic (ED) volume (EDV) - end-systolic (ES) volume (ESV)]/ $\mathrm{EDV} \times 100$. The $\mathrm{ED} \mathrm{fRV} / \mathrm{LV}$ volume ratio was calculated. The volume-based SI and the total right/left-volume index at the ED were calculated using the following formulas: volume-based $\mathrm{SI}=($ right atrial $\mathrm{EDV}+\mathrm{aRV} \mathrm{EDV}) /(\mathrm{fRV} \mathrm{EDV}+$ left atrial $\mathrm{EDV}+\mathrm{LV}$ $\mathrm{EDV})$; and total right/left-volume index $=($ right atrial $\mathrm{EDV}+\mathrm{RV}$ $\mathrm{EDV}) /($ left atrial EDV+LV EDV) [19]. In addition, the fRV fractions at the ES and ED phases were calculated as follows: $\mathrm{fRV}$ fraction $(\%)=[\mathrm{fRV}$ volume/total RV $(\mathrm{tRV})$ volume $] \times 100$.

In order to evaluate the reproducibility of the volumetric measurements, the volume quantification of cardiac chambers were repeated 2-8 years after the first quantification session. Any inter-measurement differences between the two sessions were calculated.

\section{Area-based CT severity index}

The ED four-chamber CT image was used to measure the areas of the cardiac chambers (Fig. 1H). The area-based SI was calculated using the following formula: area-based SI=(right atrial area $+\mathrm{aRV}$ area $) /(\mathrm{fRV}$ area+left atrial area $+\mathrm{LV}$ area $)[1,20]$.

\section{Echocardiographic severity index}

The echocardiographic findings were reviewed with regard to the Carpentier types (A, appropriate fRV volume; B, large $\mathrm{aRV}$ with the freely moving anterior leaflet; $\mathrm{C}$, severely restricted motion of the anterior leaflet potentially obstructing the RV outflow tract; D, almost entirely aRV except for a small infundibular segment) and Celermajer area-based SI (Fig. 1I).

\section{Severity grading}

The area- and volume-based CT SIs and the area-based echo- cardiographic SI were graded as previously described: grade 1, $<0.50$; grade 2, 0.50-0.99; grade 3, 1.00-1.49; grade $4,>1.50$ $[1,20]$.

\section{Data description}

The continuous variables are presented as means \pm standard deviations or medians with ranges. The categorical variables are expressed as frequencies and percentages.

\section{RESULTS}

\section{CT image quality evaluation}

With regard to the delineated tricuspid valve, grade 4 was shown in $83.3 \%$ (5/6) of the cases (Fig. 1A-C) and grade 3 in $16.7 \%(1 / 6)$. The aRV and fRV parts, therefore, could be threedimensionally separated from each other along the delineated tricuspid valve plane on the cardiac CT images (Fig. 1D). With regard to the streak artifacts, grade 4 was found in $33.3 \%(2 / 6)$ of cases and grade 3 in $66.7 \%(4 / 6)$. These artifacts were usually observed around the superior vena cava and the right atrium. The measured heterogeneity in contrast enhancement in the cardiac chambers was $49.6 \pm 39.4 \mathrm{HU}$ for the right atrium, $26.4 \pm$ $9.8 \mathrm{HU}$ for the left atrium (LA), $26.2 \pm 12.1 \mathrm{HU}$ for the aRV, $26.0 \pm 11.7 \mathrm{HU}$ for the fRV, and $19.9 \pm 6.5 \mathrm{HU}$ for the LV. In only one case, the right atrium had image noise $(126.7 \mathrm{HU})$ that was $>50 \mathrm{HU}$ (Fig. $1 \mathrm{~A}$ and B). In contrast, all other cases demonstrated image noise $<50 \mathrm{HU}$. The image quality was appropriate to sufficiently make all of the volumetric measurements (Fig. 1D-G).

\section{Volumetric CT parameters}

Volumetric CT parameters are provided in Table 1. As compared to the normal right- and left ventricular volumes in body size- and sex-matched children measured by cardiac MRI [21], the $\mathrm{RV}$ volumes measured by cardiac $\mathrm{CT}$ were enlarged in $83.3 \%$ (5/6) of the cases. In contrast, the LV volumes remained within the normal range in $83.3 \%$ (5/6) of cases. Interestingly, one patient who had undergone a central shunt and division of a patent ductus arteriosus had normal tRV volumes and increased LV volumes. With respect to the fRV fraction, the fRV volumes were greater than the aRV volumes in four cases, while the fRV volumes were smaller than the aRV volumes in two cases. Fiftypercent of patients ( $3 / 6$ cases) had an EF of the tRV that was $<50 \%$. In contrast, the LVEF was within normal range in all of the cases. The ED fRV/LV volume ratios were $>2.0$ in 50.0\% (3/6) of the cases, between 1.0 and 2.0 in $33 \%$ (2/6), and 0.11 in $16.7 \%(1 / 6)$. The ED total right/left-volume indices were $>4.0$ in $66.7 \%(4 / 6)$ of the cases, between 2.0 and 4.0 in $16.7 \%(1 / 6)$, and between 1.0 and 2.0 in $16.7 \%(1 / 6)$.

The inter-measurement differences in the cardiac chamber 

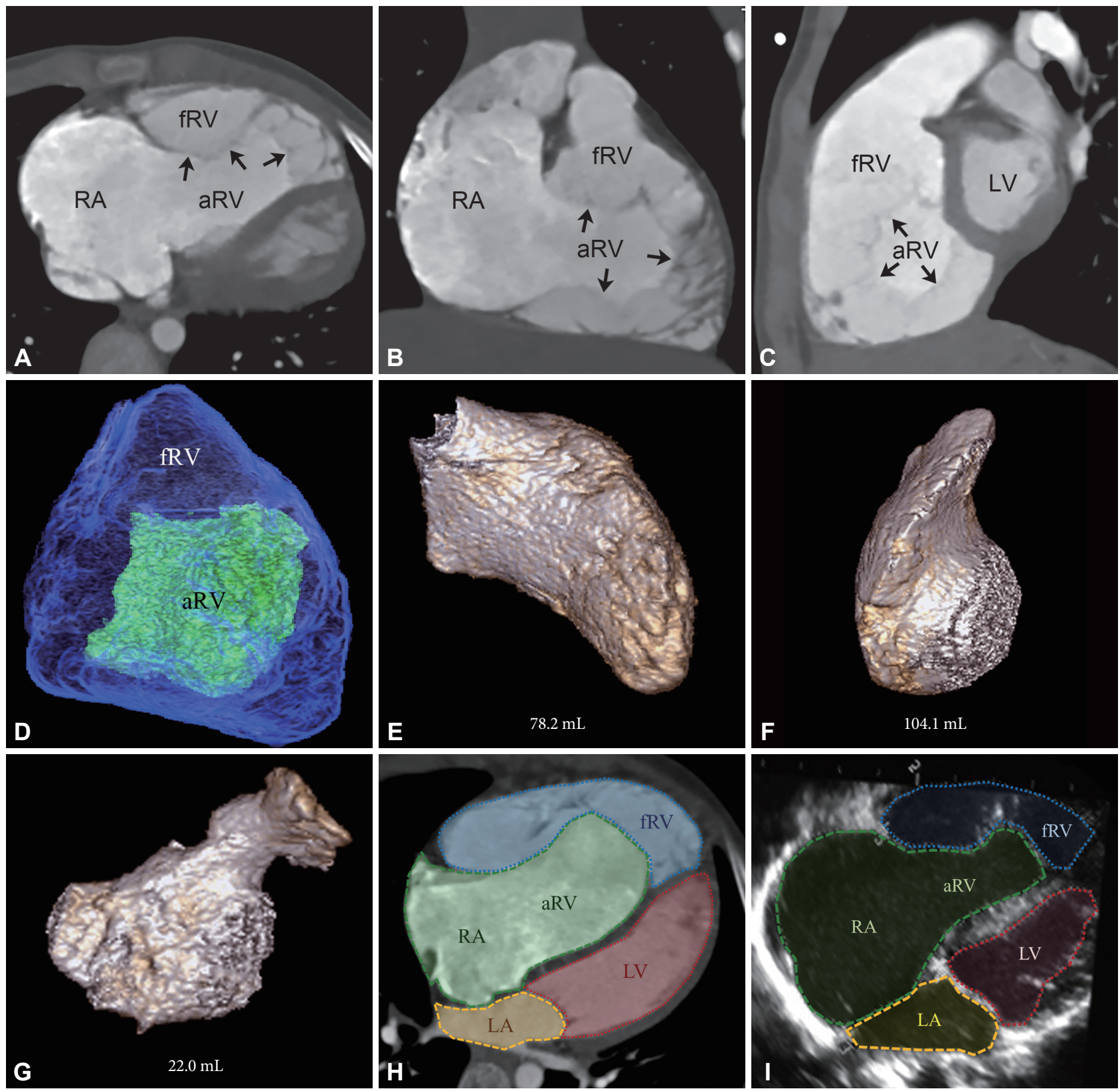

Fig. 1. A 10-year-old boy with unrepaired Ebstein anomaly. On axial (A), coronal (B), and sagittal (C) reformatted CT images, the malformed tricuspid valve plane (arrows) is almost completely delineated (grade 4). Residue from poorly diluted contrast agent is noted in the dilated RA, which caused a mild degree of streak artifacts (grade 3 ) and heterogeneous contrast enhancement (126.7 HU) (A and B). Color-coded frontal volume-rendered CT image (D) shows the aRV in green, and the fRV in blue. The tRV (305.8 mL) was divided into the aRV (83.8 $\mathrm{mL})$ and the $\mathrm{fRV}(222.0 \mathrm{~mL})$ along the malformed tricuspid valve. Based on these measurements, the fRV fraction was $72.6 \%$. The frontal volume-rendered CT images (E-G) demonstrate the LV volume $(78.2 \mathrm{~mL})$, the RA volume $(104.1 \mathrm{~mL})$, and the LA volume $(22.0 \mathrm{~mL})$. ED fourchamber CT image $(\mathrm{H})$ shows the color-coded areas of each cardiac chamber used to calculate the area-based CT SI (0.8), which is analogous to the echocardiographic area-based Celermajer SI (1.1) obtained from ED four-chamber echocardiographic image (I). aRV: atrialized right ventricle, fRV: functional right ventricle, LA: left atrium, LV: left ventricle, RA: right atrium, tRV: total right ventricle, HU: Hounsfield units, ED: end-diastolic, SI: severity index.

volumes were small, as follows, which suggested high reproducibility of the approach: $1.3 \pm 0.9 \%$ for the aRV, $1.0 \pm 0.6 \%$ for the fRV, $0.8 \pm 0.5 \%$ for the $\mathrm{LV}, 1.6 \pm 1.0 \%$ for the RA, and $1.5 \pm 0.8 \%$ for the LA.

\section{Comparing echocardiographic and CT severity indices}

The average values were similar in all three indices in the range of 0.9-1.0 (Table 2). The differences were as follows: $0.3 \pm 0.5$ (median 0.1, range 0.1-1.3) between the echocardiographic Celermajer area-based SI and the area-based CT SI; $0.3 \pm 0.4$ (median 0.1, range $0.0-1.0$ ) between the echocardio- 
Table 1. Volumetric CT parameters of cardiac chambers

\begin{tabular}{lc}
\hline & Ebstein anomaly $(\mathrm{n}=6)$ \\
\hline aRV ESVi $\left(\mathrm{mL} / \mathrm{m}^{2}\right)$ & $29.3 \pm 12.8$ \\
fRV ESVi $\left(\mathrm{mL} / \mathrm{m}^{2}\right)$ & $66.1 \pm 53.7$ \\
tRV ESVi $\left(\mathrm{mL} / \mathrm{m}^{2}\right)$ & $95.4 \pm 54.0$ \\
aRV EDVi $\left(\mathrm{mL} / \mathrm{m}^{2}\right)$ & $57.2 \pm 33.1$ \\
fRV EDVi $\left(\mathrm{mL} / \mathrm{m}^{2}\right)$ & $126.2 \pm 89.9$ \\
tRV EDVi $\left(\mathrm{mL} / \mathrm{m}^{2}\right)$ & $183.4 \pm 97.7$ \\
LV ESVi $\left(\mathrm{mL} / \mathrm{m}^{2}\right)$ & $30.2 \pm 11.4$ \\
LV EDVi $\left(\mathrm{mL} / \mathrm{m}^{2}\right)$ & $70.6 \pm 23.6$ \\
RA ESVi $\left(\mathrm{mL} / \mathrm{m}^{2}\right)$ & $171.6 \pm 32.7$ \\
RA EDVi $\left(\mathrm{mL} / \mathrm{m}^{2}\right)$ & $97.5 \pm 26.0$ \\
LA ESVi $\left(\mathrm{mL} / \mathrm{m}^{2}\right)$ & $33.8 \pm 5.5$ \\
LA EDVi $\left(\mathrm{mL} / \mathrm{m}^{2}\right)$ & $19.6 \pm 12.9$ \\
ES fRV fraction $(\%)$ & $60.8 \pm 22.4$ \\
ED fRV fraction (\%) & $61.5 \pm 24.8$ \\
tRV ejection fraction $(\%)$ & $46.0 \pm 10.7$ \\
LV ejection fraction (\%) & $57.6 \pm 2.7$ \\
ED fRV/LV volume ratio & $2.0 \pm 1.4$ \\
ED volume-based severity index & $0.9 \pm 0.7$ \\
ED total right/left-volume index & $3.5 \pm 1.5$ \\
\hline
\end{tabular}

Data are presented as means \pm standard deviations. aRV: atrialized right ventricle, ED: end-diastolic, EDVi: indexed end-diastolic volume, ES: end-systolic, ESVi: indexed ES volume, fRV: functional right ventricle, $L A$ : left atrium, LV: left ventricle, RA: right atrium, tRV: total right ventricle

Table 2. Echocardiographic and CT SIs measured in end-diastole

\begin{tabular}{lcc}
\hline & Echocardiography $(\mathrm{n}=6)$ & $\mathrm{CT}(\mathrm{n}=6)$ \\
\hline Area-based SI & $0.9 \pm 0.3$ & $1.0 \pm 0.8$ \\
Volume-based SI & N/A & $0.9 \pm 0.7$ \\
\hline
\end{tabular}

Data are presented as means \pm standard deviations. SI: severity index

graphic Celermajer area-based SI and the volume-based CT SI; and $0.2 \pm 0.1$ (median 0.1 , range $0.1-0.3$ ) between the area-based CT SI and the volume-based CT SI. The severity grades were concordant in $50 \%$ of the cases (3/6) between the echocardiographic Celermajer area-based SI and the area-based or volumebased CT SI (Table 3). A higher concordance rate of $83.3 \%$ (5/6) in the severity grades was found between the area-based CT SI and the volume-based CT SIs (Table 3). With regard to the echocardiographic Carpentier types, one case had type A, three had type $\mathrm{B}$, one had type $\mathrm{C}$, and one type $\mathrm{D}$. The Carpentier types $\mathrm{A}-\mathrm{C}$ tended to be proportional to the echocardiographic and CT SIs. However, the Carpentier type D showed values between types B and C in the echocardiographic and CT SIs (Table 4).

\section{DISCUSSION}

This study demonstrates the feasibility of volumetric severity assessment using $3 \mathrm{D}$ isotropic cardiac $\mathrm{CT}$ in children with $\mathrm{Eb}$ -
Table 3. Comparisons between echocardiographic and CT Sls measured in end-diastole

\begin{tabular}{cccc}
\hline $\begin{array}{c}\text { Case } \\
\text { number }(\mathrm{n}=6)\end{array}$ & $\begin{array}{c}\text { Echocardiographic } \\
\text { SI }\end{array}$ & $\begin{array}{c}\text { Area-based } \\
\text { CT SI }\end{array}$ & $\begin{array}{c}\text { Volume-based } \\
\text { CT SI }\end{array}$ \\
\hline 1 & 3 & 2 & 2 \\
2 & 2 & 2 & 2 \\
3 & 2 & 2 & 2 \\
4 & 1 & 2 & 1 \\
5 & 3 & 4 & 4 \\
6 & 2 & 2 & 2 \\
\hline
\end{tabular}

SI: severity index

Table 4. Echocardiographic and CT SIs measured at end-diastole by the echocardiographic Carpentier types

\begin{tabular}{cccc}
\hline $\begin{array}{c}\text { Carpentier } \\
\text { type }\end{array}$ & $\begin{array}{c}\text { Echocardiographic } \\
\text { SI }\end{array}$ & $\begin{array}{c}\text { Area-based } \\
\text { CT SI }\end{array}$ & $\begin{array}{c}\text { Volume-based } \\
\text { CT SI }\end{array}$ \\
\hline A $(n=1)$ & 0.4 & 0.6 & 0.4 \\
B $(n=3)$ & 0.8 & 0.7 & 0.6 \\
C $(n=1)$ & 1.3 & 2.6 & 2.3 \\
D $(n=1)$ & 1.0 & 0.9 & 0.9 \\
\hline
\end{tabular}

SI: severity index

stein anomaly. This method of analysis also allows for measurement of the functional parameters, including the aRV volume, fRV volume, fRV fraction, fRV/LV ratio, volume-based SI, and total right/left-volume index. Classically, 2D cardiac MRI has been considered advantageous over echocardiography for quantifying RV volume and function in Ebstein anomaly [22]. However, the accurate delineation of the tricuspid valve plane in Ebstein anomaly using time-consuming manual tracing [23] or geometric modeling [5] still appears to be problematic using 2D cardiac MRI. A 3D approach may avoid the potential errors derived from $2 \mathrm{D}$ imaging, $2 \mathrm{D}$ segmentation, and geometric assumption [4-6]. Other researchers have suggested that volumetric quantification using a 3D imaging modality may expedite a better understanding of the relationship between the RV status and disease severity in Ebstein anomaly [4,5]. In particular, the use of 3D threshold-based segmentation could eliminate the time-consuming and reader-dependent manual endocardial tracings for ventricular volumetry as demonstrated in previous studies $[7,9]$. The threshold-based approach was reported to show high reproducibility with an inter-measurement difference of $1.0 \pm 1.1 \%$, and intraclass correlation coefficient of 1.0 [24]. We also found that the inter-measurement volume differences were $<2.0 \%$ in all cardiac chambers.

Achieving high image quality with cardiac $\mathrm{CT}$ is a prerequisite to the success of ventricular volume quantification using the 3D threshold-based approach. In Ebstein anomaly, the delineation of the malformed tricuspid valve plane is quite challenging. Previously, the valve plane was too simplified on imaging. Due to its poor definition and limited spatial resolution on 2D MRI, 
the valve plane was portrayed as a straight line connecting the free wall attachment of the anterior tricuspid valve leaflet at the level of the annulus and the septal attachment of the apically displaced leaflet. These oversimplifications may have led to overestimation of the fRV volumes and underestimation of the aRV volumes $[4,23]$. In contrast, the variously malformed tricuspid valve planes could be delineated in $>50 \%$ of all cases and by $>75 \%$ in most cases using 3D CT images in this study. With 3D imaging, the entire valve plane could be segmented by bridging the short gaps between the delineated valve leaflets. Streak artifacts from undiluted contrast agent, which potentially complicate 3D threshold-based segmentation, could be reduced by using optimized intravenous contrast injection protocols. It is challenging to produce homogeneous contrast enhancement in the right atrium [25]. However, using our protocol, there was only one case in which the attenuation heterogeneity was $>50 \mathrm{HU}$ in the right atrium due to residue from poorly diluted contrast.

The average values of the echocardiographic Celermajer area-based SI, the area-based CT SI, and the volume-based CT SI measured at the end-diastolic phase were similar (0.9-1.0) across cases. However, the individual differences were quite variable in the range of $0.0-1.3$. Among them, smaller differences and a higher concordance rate in severity grades were obtained between the area-based CT SI and the volume-based CT SI. This result might be attributed to the use of the same imaging modality. These echocardiographic and CT SIs were proportionate to the echocardiographically-defined Carpentier type in types A-C. Theoretically, the fRV area is subject to underestimation, because the majority of the fRV is out-of-plane on a four-chamber view. In fact, the fRV size appears generally larger on 3D imaging than it does on 2D imaging [26]. Therefore, there is a need for better severity assessment using the $3 \mathrm{D}$ approach to determine optimal surgical timing and the appropriate surgical procedure in Ebstein anomaly given its diverse regional patterns. To date, the role of cardiac CT in assessing Ebstein anomaly has been limited to an anecdotal description of the anomaly, or to the associated coronary artery anomaly $[27,28]$. In this respect, we believe that our study expands the role of cardiac CT in assessing Ebstein anomaly and volumetric severity assessment using 3D cardiac CT. 3D imaging may contribute to improved risk stratification in Ebstein anomaly. However, further studies are needed to substantiate its clinical value.

The ED fRV/LV volume ratio of 2.0 in this study was lower than that (median 2.5) in a previous study [23]. This discrepancy might be attributable to the fact that we included pediatric patients, who have small fRV volumes compared to those of adults. Adult patients with Ebstein anomalies have an fRV fraction that ranges $68-88 \%$, compared to $61.5 \%$ in this study from pediatric patients $[4,19,23,29]$. In contrast, the ED total right/ left-volume index of 3.5 in this study was higher than that (2.6) previously described [19]. This difference may be attributed to our inclusion of patients with more severely dilated RVs: indexed aRV EDV $57.2 \mathrm{~mL} / \mathrm{m}^{2}$ (this study) vs. $52-53 \mathrm{~mL} / \mathrm{m}^{2}$ (the previous study), indexed fRV EDV $126.2 \mathrm{~mL} / \mathrm{m}^{2}$ (this study) vs. $115-118 \mathrm{~mL} / \mathrm{m}^{2}$ (the previous study). The ED total right/leftvolume index has been suggested to be a better predictor of heart failure than are the SI and the ED fRV/LV volume ratio in Ebstein anomaly [19].

This study has several limitations. First, we included a small number of patients, which only allowed for descriptive statistics. Regardless, this study appears to demonstrate the technical feasibility of volumetric severity assessment of Ebstein anomaly using $3 \mathrm{D}$ cardiac $\mathrm{CT}$ data. A second limitation is that the current reference standards for cardiac MRI studies were not available for the comparison with the results of cardiac CT. However, performing cardiac CT and MRI in the same patients is likely very difficult, or impractical in real clinical practice. Still, a future prospective study may overcome this limitation. Another limitation is that this study may have been subject to selection bias (to include patients with more severe Ebstein anomaly), because cardiac CT is not yet a standard imaging modality at our institution. Fourth, a practical limitation to this approach is that it takes a relatively long time to process and segment all of the cardiac chambers. More experience with this approach, however, may reduce the processing time [30,31].

In conclusion, volumetric severity assessment using 3D cardiac CT is technically feasible without any geometric assumptions of the malformed tricuspid valve in Ebstein anomaly. This approach may be useful to characterize the diverse phenotypes of Ebstein anomaly. However, further research is needed to substantiate the preliminary results of this study.

\section{Conflicts of Interest}

The author has no potential conflicts of interest to disclose.

\section{REFERENCES}

1. Negoi RI, Ispas AT, Ghiorghiu I, Filipoiu F, Negoi I, Hostiuc M, et al. Complex Ebstein's malformation: defining preoperative cardiac anatomy and function. J Card Surg 2013;28:70-81.

2. Sirivella S, Gielchinsky I. Surgery of the Ebstein's anomaly: early and late outcomes. J Card Surg 2011;26:227-233.

3. Warnes CA, Williams RG, Bashore TM, Child JS, Connolly HM, Dearani JA, et al. ACC/AHA 2008 guidelines for the management of adults with congenital heart disease: a report of the American College of Cardiology/ American Heart Association Task Force on practice guidelines (writing committee to develop guidelines on the management of adults with congenital heart disease). Developed in collaboration with the American Society of Echocardiography, Heart Rhythm Society, International Society for adult congenital heart disease, Society for Cardiovascular Angiography and Interventions, and Society of Thoracic Surgeons. J Am Coll Cardiol 2008;52:e143-e263.

4. Yalonetsky S, Tobler D, Greutmann M, Crean AM, Wintersperger BJ, Nguyen ET, et al. Cardiac magnetic resonance imaging and the assessment of Ebstein anomaly in adults. Am J Cardiol 2011;107:767-773. 
5. Lee CM, Sheehan FH, Bouzas B, Chen SS, Gatzoulis MA, Kilner PJ. The shape and function of the right ventricle in Ebstein's anomaly. Int J Cardiol 2013;167:704-710.

6. Liu X, Zhang Q, Yang ZG, Guo YK, Shi K, Xu HY, et al. Morphologic and functional abnormalities in patients with Ebstein's anomaly with cardiac magnetic resonance imaging: correlation with tricuspid regurgitation. Eur J Radiol 2016;85:1601-1606.

7. Goo HW. Comparison between three-dimensional navigator-gated wholeheart MRI and two-dimensional cine MRI in quantifying ventricular volumes. Korean J Radiol 2018;19:704-714.

8. Goo HW. State-of-the-art CT imaging techniques for congenital heart disease. Korean J Radiol 2010;11:4-18.

9. Goo HW. Semiautomatic three-dimensional threshold-based cardiac computed tomography ventricular volumetry in repaired tetralogy of Fallot: comparison with cardiac magnetic resonance imaging. Korean J Radiol 2019;20:102-113.

10. Lee E, Goo HW, Lee JY. Age- and gender-specific estimates of cumulative CT dose over 5 years using real radiation dose tracking data in children. Pediatr Radiol 2015;45:1282-1292.

11. Hui PKT, Goo HW, Du J, Ip JJK, Kanzaki S, Kim YJ, et al. Asian consortium on radiation dose of pediatric cardiac CT (ASCI-REDCARD). Pediatr Radiol 2017;47:899-910.

12. Hong SH, Goo HW, Maeda E, Choo KS, Tsai IC: Asian Society of Cardiovascular Imaging Congenital Heart Disease Study Group. User-friendly vendor-specific guideline for pediatric cardiothoracic computed tomography provided by the Asian Society of Cardiovascular Imaging Congenital Heart Disease Study Group: Part 1. Imaging techniques. Korean J Radiol 2019;20:190-204.

13. Goo HW, Suh DS. Tube current reduction in pediatric non-ECG-gated heart CT by combined tube current modulation. Pediatr Radiol 2006;36: 344-351.

14. Goo HW. Cardiac MDCT in children: CT technology overview and interpretation. Radiol Clin North Am 2011;49:997-1010.

15. Goo HW. Comparison of chest pain protocols for electrocardiographygated dual-source cardiothoracic CT in children and adults: the effect of tube current saturation on radiation dose reduction. Korean J Radiol 2018;19:23-31.

16. Goo HW. Individualized volume CT dose index determined by cross-sectional area and mean density of the body to achieve uniform image noise of contrast-enhanced pediatric chest CT obtained at variable $\mathrm{kV}$ levels and with combined tube current modulation. Pediatr Radiol 2011;41:839847.

17. Goo HW. Combined prospectively electrocardiography- and respiratorytriggered sequential cardiac computed tomography in free-breathing children: success rate and image quality. Pediatr Radiol 2018;48:923-931.
18. Goo HW. CT radiation dose optimization and estimation: an update for radiologists. Korean J Radiol 2012;13:1-11.

19. Hösch O, Sohns JM, Nguyen TT, Lauerer P, Rosenberg C, Kowallick JT, et al. The total right/left-volume index: a new and simplified cardiac magnetic resonance measure to evaluate the severity of Ebstein anomaly of the tricuspid valve: a comparison with heart failure markers from various modalities. Circ Cardiovasc Imaging 2014;7:601-609.

20. Celermajer DS, Cullen S, Sullivan ID, Spiegelhalter DJ, Wyse RK, Deanfield JE. Outcome in neonates with Ebstein's anomaly. J Am Coll Cardiol 1992;19:1041-1046.

21. Buechel EV, Kaiser T, Jackson C, Schmitz A, Kellenberger CJ. Normal right- and left ventricular volumes and myocardial mass in children measured by steady state free precession cardiovascular magnetic resonance. J Cardiovasc Magn Reson 2009;11:19.

22. Attenhofer Jost CH, Edmister WD, Julsrud PR, Dearani JA, Savas Tepe M, Warnes CA, et al. Prospective comparison of echocardiography versus cardiac magnetic resonance imaging in patients with Ebstein's anomaly. Int J Cardiovasc Imaging 2012;28:1147-1159.

23. Fratz S, Janello C, Müller D, Seligmann M, Meierhofer C, Schuster T, et al. The functional right ventricle and tricuspid regurgitation in Ebstein's anomaly. Int J Cardiol 2013;167:258-261.

24. Goo HW, Park SH. Computed tomography-based ventricular volumes and morphometric parameters for deciding the treatment strategy in children with a hypoplastic left ventricle: preliminary results. Korean J Radiol 2018;19:1042-1052.

25. Goo HW. Haemodynamic findings on cardiac CT in children with congenital heart disease. Pediatr Radiol 2011;41:250-261.

26. Booker OJ, Nanda NC. Echocardiographic assessment of Ebstein's anomaly. Echocardiography 2015;32 Suppl 2:S177-S188.

27. Aggarwala G, Thompson B, van Beek E, Jagasia D. Multislice computed tomography angiography of Ebstein anomaly and anomalous coronary artery. J Cardiovasc Comput Tomogr 2007;1:168-169.

28. Zikria JF, Dillon EH, Epstein NF. Common CTA features of Ebstein anomaly in a middle-aged woman with a heart murmur and dyspnea on exertion. J Cardiovasc Comput Tomogr 2012;6:431-432.

29. Tobler D, Yalonetsky S, Crean AM, Granton JT, Burchill L, Silversides CK, et al. Right heart characteristics and exercise parameters in adults with Ebstein anomaly: new perspectives from cardiac magnetic resonance imaging studies. Int J Cardiol 2013;165:146-150.

30. Ngo TA, Lu Z, Carneiro G. Combining deep learning and level set for the automated segmentation of the left ventricle of the heart from cardiac cine magnetic resonance. Med Image Anal 2017;35:159-171.

31. Dou Q, Yu L, Chen H, Jin Y, Yang X, Qin J, et al. 3D deeply supervised network for automated segmentation of volumetric medical images. Med Image Anal 2017;41:40-54. 